\title{
Studies of food intakes of schoolchildren
}

By Ailsa Melton and Betty Y. Winstanley, Department of Clinical Epidemiology and Social Medicine, St Thomas's Hospital Medical School, London, $S E_{\mathbf{I}}$

Text for publication not received.

\section{Flavours of dairy products}

By J. D. S. GouldeN, National Institute for Research in Dairying, Shinfield, Reading $R G_{2} 9 A T$

\section{Introduction}

From the aspect of flavour, dairy products range from milk through mildlyflavoured butter and yoghourt to the highly-flavoured cheeses. Bland products such as milk and cream have little flavour of their own and most of the flavour problems associated with such products are those caused by the presence of undesirable offflavours. These undesirable flavours can arise from feedstuffs eaten by the cow, be produced by staling or induced at various stages in the handling or processing. Since flavours are caused by trace components, sour milk will differ nutritionally very little from fresh milk, even though the flavour may be objectionable. The flavours of the various strong cheeses are produced as a result of a series of operations during manufacture, followed by storage under conditions which encourage the growth of bacteria. The importance of flavour is demonstrated by the differing prices of Cheddar cheeses with the same gross chemical composition and texture but with differing flavours.

As for other foods, flavour is a complex sensation with contributions from odour, taste and texture. Texture or 'mouth-feel' is relatively more important for bland products where the main flavour consideration is that there shall be practically no odour. Odour and taste are difficult to separate since they are linked together physiologically. Milk collected from a farm is rejected if any undesirable odour can be detected by the nose and a cheese grader assesses the flavour of cheese solely from its odour.

When we consider the possible use of physical and chemical methods for flavour assessment, we are impressed by both the sensitivity and the selectivity of the nose. A human nose can detect a few hundred molecules of an odorous compound which may be present only at the parts per million level in the presence of the major non-odorous constituents of the food. Table I shows the detectability limits for three different chemical compounds and demonstrates large differences between the values for the nose and for two different detectors frequently used in gas chromatographs. The gas-chromatograph data were obtained by Mrs H. Robinson in our 